\title{
Safety and immunogenicity of rabies pre- and post- exposure intradermal regimens using Abhayrab, a purified vero cell rabies vaccine (PVRV) produced in India in healthy volunteers: towards greater affordability of rabies prophylaxis
}

\author{
Rio L. Magpantay ${ }^{\mathrm{a}}$, Nancy Bermal ${ }^{\mathrm{b}}$, Plebeian Medina $^{\mathrm{b}}$, Beatriz P. Quiambao ${ }^{\mathrm{b}}$ \\ ${ }^{a}$ Department of Health, Centre for Health Development, Maimpis, San Fernando, Pampanga 2000; \\ ${ }^{b}$ Department of Health, Research Institute for Tropical Medicine, FCC, Alabang, Muntinlupa, Metro \\ Manila 1770, Philippines
}

\begin{abstract}
Background: Rabies is $100 \%$ fatal, but is preventable. However, due to the high cost of vaccines and immunoglobulins, as well as their limited availability, many dog-bite victims in the Philippines do not complete the required PEP regimen. This concern may be addressed by the introduction of various new brands of affordable rabies vaccines and biologicals for use with the ID regimen.

Objective: Study the safety and immunogenicity of rabies pre- and post-exposure intradermal regimens using Abhayrab, a purified vero cell rabies vaccine (PVRV) produced by the human biological institute of Ooty India in healthy volunteers.

Methods: In a randomized, single blind, unicentric trial, 149 healthy volunteers ( 5 - 50 years in age) were recruited to the study. The subjects were then randomized into two groups. Group 1 (pre-exposure) (73 subjects) followed the standard ID regimen using a dose of $0.1 \mathrm{~mL}$ PVRV (Abhayrab) given intradermally on either deltoid on days 0,7 and 28. Group 2 (post-exposure) (76 Subjects) followed the modified Thai Red Cross ID regimen (2-2-2-0-2) using a dose of $0.1 \mathrm{~mL}$ PVRV (Abhayrab) each given intradermally on both deltoids on days $0,3,7$, and 28. Following administration of vaccine, subjects were observed closely for 30 minutes at the site for adverse reactions. Four $\mathrm{ml}$ of blood from each subject was drawn on days 0,14 , and 28 for anti-rabies antibody titres. Safety and immunogenicity were assessed through follow-up of adverse events and anti rabies antibody response, respectively.

Results: Eventually, 120 subjects, 60 in each group, completed the day 28 ID immunization. All the 120 subjects demonstrated seroconversions with antibody titers greater than the WHO recommended cut-off level of $0.5 \mathrm{IU} / \mathrm{mL}$ on days 14 and 28. The GMC values for Group 1 were $3.30 \mathrm{IU} / \mathrm{ml}$ and $4.37 \mathrm{IU} / \mathrm{mL}$ on days 14 and 28 , respectively, while those of Group 2 were $3.73 \mathrm{IU} / \mathrm{mL}$ and $4.82 \mathrm{IU} / \mathrm{mL}$, respectively. These GMC values were within their 95\% CIs. Only a few mild adverse events were observed with no incidences of moderate or severe events.
\end{abstract}

Conclusion: Abhayrab is a safe and immunogenic rabies vaccine when administered intradermally.

Keywords: Human rabies, PVRV, Intradermal, post-exposure treatment, RFFIT

Rabies is one of the major public health concerns in the Philippines. It is estimated that 200 to 300 Filipinos die every year of rabies [Phil. Department of Health- DOH]. Of the estimated 150,000 dog-bite consultations [Phil. DOH] in the entire country each year, at least 60,000 are estimated to be severe bites for which complete post-exposure prophylaxis (PEP) is indicated. A review of single point data from the 
Research Institute for Tropical Medicine (RITM) OutPatient Department in the Philippines revealed that of 14,449 consults for dog bites, $98 \%$ were prescribed PEP [RITM Public Library of Science 2008].

Since rabies is known to be $100 \%$ fatal once symptoms have set in, prevention through PEP is the only proven means to save rabies-infected patients. Considering the gravity of rabies, and that dog vaccination and control measures for dogs in the country are far from ideal, Pre-exposure and PEP are of utmost importance. However, it is not surprising to find that many Filipinos bitten by suspected rabid animals do not complete the required PEP regimen. This is mainly due to the high cost of PEP vaccines and immunoglobulins, as well as to their limited availability [1].

The Philippine Department of Health (DOH), in partnership with other government agencies, particularly the Department of Agriculture, aims to eliminate rabies and declare the Philippines rabiesfree by 2020. One of DOH's major strategies for rabies elimination is the provision of affordable rabies PEP through the more than 200 animal bite treatment centers distributed all over the country. In order to do this, a reliable supply of affordable vaccines and the use of the economical reduced-dose multi-site intradermal (ID) schedule must be ensured. The twosite intradermal regimen of rabies vaccination has been well established in Thailand and in the Philippines since 1984 and 1993, respectively, and it has offered patients significant amounts of savings of up to $80 \%$ compared with the application of the standard full-course regimen using the same vaccine [WHO Recommendation on PEP 24 June 2009]. The WHO Expert Committee on Rabies has recommended the use of the ID regimen for rabies PEP since 1991 [2, 3].

As there are now a growing number of rabies vaccine manufacturers able to supply more affordable quality rabies vaccines and biologicals, the introduction of various new brands in the Philippine market is expected to benefit the general public through competition, the direct reduction in cost of PEP. However, such new products should be proven first to be safe, immunogenic and efficacious through the conduct of clinical trials.

A vaccine that is considered to be an affordable alternative was recently introduced in the Philippines, Abhayrab, is a purified vero cell rabies vaccine (PVRV) manufactured in India by Human Biologicals Institute. Since its introduction in the Philippines, it has been used in both pre-exposure and post-exposure regimens. The vaccine has previously been proven safe and immunogenic by the intramuscular regimen in India, where it has been used extensively since 2000 [4-6]. In 2006, based on an independently conducted ID safety and immunogenicity study, the Indian government permitted the ID use of four types of rabies vaccines, including Abhayrab, to significantly bring down the cost and ensure the provision of effective rabies PEP, replacing the use of the low quality and dangerous semple vaccine [7].

This randomized controlled clinical trial documented the safety and immunogenicity of PVRV (Abhayrab) when used in both pre-exposure and postexposure rabies prophylaxes by following the Thai Red Cross ID regimen. In this study, we conducted the trial in healthy volunteers in whom safety was evaluated through clinical observations for local and systemic reactions. Immunogenicity was likewise determined through RFFIT assays of rabies neutralizing antibodies.

\section{Materials and methods \\ Ethical approval and GCP}

Approval to conduct the study was obtained from the Ethics Review Committee of the Philippine Department of Health (DOH) prior to the start of the study. The study was conducted following the international guidelines on Good Clinical Practice.

\section{Study location}

The trial was conducted at the Reference Laboratory of the Collaborating Centre for Disease Prevention and Control, Department of Health (DOH) Centre for Health Development for Central Luzon, in the city of San Fernando, Pampanga. The determination of the antibody responses of the subjects was done at the Rabies Research Laboratory of the Research Institute for Tropical Medicine, Department of Health, Alabang, Muntinlupa City.

Pampanga is among the 10 Philippine provinces with the most number of animal rabies. The Provincial Health Office (PHO) reported 2,033 cases of animal bites (27.52 per 100,000 population) in 2008. In 2007, there were five reported cases of human deaths due to rabies in the province.

\section{Sample size}

The sample size was computed based on the formula for the estimation of a single proportion with allowance for dropouts. 


\section{Inclusion and grouping of volunteer subjects}

All subjects were healthy volunteers recruited from the city of San Fernando, Pampanga between September and November 2006. Written informed consent was obtained from all the subjects or from the legal guardians for subjects below 18 years of age. The inclusion/exclusion criteria for both groups were the same. The subjects were all healthy volunteers from 5 to 50 years of age. The subject exclusion criteria were: had previously received antirabies vaccination; pregnant or lactating; blood extraction was judged to be difficult; had no permanent address or had plans of traveling within the next three months; unable to comply with the visit schedule of the protocol; enrolled in another clinical trial; with signs of rabies; receiving immunosuppressive therapy or cytotoxic drugs; with prior history of dog/cat bite where the biting animal died or outcome is unknown; have received blood and/or plasma transfusion within the past three months; with known chronic illness; who received any vaccine within the previous 30 days (except tetanus toxoid and tetanus immune globulin); and with known allergy to the vaccine. Upon enrollment, the subjects underwent detailed history taking and thorough physical examination.

The subjects were then randomized into two groups. Group 1 (Pre-exposure) followed the standard ID regimen using a dose of $0.1 \mathrm{~mL}$ PVRV (Abhayrab) given intradermally on either deltoid on days 0,7 and 28. Group 2 (Post-exposure) followed the modified Thai Red Cross ID regimen (2-2-2-0-2) using a dose of $0.1 \mathrm{ml}$ PVRV (Abhayrab) each given intradermally on both deltoids on days $0,3,7$, and 28 . Randomization was performed by taking one sealed envelope containing the randomly arranged grouping code. A nurse recorded the number allocated to the subject and the assigned code.

\section{Vaccine and vaccination}

The trial vaccine used, Abhayrab, is a purified vero cell based beta-propiolactone inactivated rabies vaccine manufactured by Human Biologicals Institute. The vaccines were from a common commercial lot (lot no: AYB1106) manufactured in May 2006. Each single dose vial contained lyophilized rabies antigens of strain L. Pasteur-2061/Vero with an NIH test potency of $5.56 \mathrm{IU}$. This was reconstituted to $0.5 \mathrm{~mL}$ with $0.9 \%$ sodium Chloride prior to injection. Each vial preparation also contained $0.015 \%$ thiomersol, maltose, and human serum albumin. The reconstituted vaccine from which each $0.1 \mathrm{~mL}$ ID dose was withdrawn was kept refrigerated, and was discarded 8 hours after reconstitution even if the entire $0.5 \mathrm{~mL}$ content had not been fully consumed. A nurse administered the vaccine intradermally following the WHO recommendation [8]. A one-milliliter syringe fitted with a 26-gauge needle was used in administering the vaccine.

\section{Vaccination safety evaluation}

After vaccination, the subjects were asked to stay for 30 minutes and were evaluated for any occurrence of adverse reactions/events as defined in Table $\mathbf{1}$.

The subjects were asked to return for subsequent doses of the vaccine and for blood extraction. At each visit, the subjects were asked about any adverse reactions they personally noted. Efforts were made to ensure that the subjects returned for follow-up doses and examination, including conveying reminders by phone and/or home visits.

Table 1. The severity scale used for evaluating the adverse reactions observed after the ID vaccination.

\begin{tabular}{|c|c|c|c|}
\hline Local reactions & $1=$ Mild & 2 = Moderate & $3=$ Severe \\
\hline Pain & $\begin{array}{l}\text { Reacts when } \\
\text { site is touched }\end{array}$ & Cries when site is touched & $\begin{array}{l}\text { Cries when limb is } \\
\text { moved }\end{array}$ \\
\hline $\begin{array}{l}\text { Redness, swelling } \\
\text { Induration/unsolicited } \\
\text { local reactions }\end{array}$ & $\begin{array}{l}\text { Largest diameter of } \\
\text { the reaction }<2.5 \mathrm{~cm}\end{array}$ & $\begin{array}{l}\text { Largest diameter of the } \\
\text { reaction between } 2.5-5.0 \mathrm{~cm}\end{array}$ & $\begin{array}{l}\text { Largest diameter of } \\
\text { the reaction }>5.0 \mathrm{~cm}\end{array}$ \\
\hline Itchiness & Easily tolerated & $\begin{array}{l}\text { Sufficiently discomforting to } \\
\text { interfere with daily activity }\end{array}$ & $\begin{array}{l}\text { Preventing normal } \\
\text { daily activity }\end{array}$ \\
\hline \multicolumn{4}{|l|}{ Systemic reactions } \\
\hline Fever & $37.5-38.0^{\circ} \mathrm{C}$ & $38.1-39.5^{\circ} \mathrm{C}$ & $\geq 39.6^{\circ} \mathrm{C}$ \\
\hline $\begin{array}{l}\text { Malaise, headache, } \\
\text { rash, myalgia, GI upset }\end{array}$ & Easily tolerated & $\begin{array}{l}\text { Sufficiently discomforting } \\
\text { To interfere with daily activity }\end{array}$ & $\begin{array}{l}\text { Preventing normal } \\
\text { daily activity }\end{array}$ \\
\hline
\end{tabular}




\section{Serum sample collection and antibody assay}

Four milliliters of blood from each subject were drawn on days 0 (pre-vaccination sample), 14 and 28. The schedule for blood sampling in some subjects deviated by \pm 1 day for the day 14 samples and \pm 3 days for day 28 samples.

Blood samples were drawn by a medical technologist using plain vacutainer tubes. A label was affixed onto the vacutainer tube immediately prior to blood sample drawing. The collected blood samples were left at room temperature for 30 minutes to two hours for clotting and then centrifuged at $3000 \mathrm{rpm}$ for 5 to 10 minutes to separate the sera. The serum samples were aliquoted and coded, and kept frozen at -20 to $-80^{\circ} \mathrm{C}$ throughout the study period. The temperatures of the freezers were monitored and documented during the entire study period. The coded serum samples were dispatched to the Rabies Research Laboratory of the Research Institute for Tropical Medicine, Department of Health, Alabang, Muntinlupa City for determination of neutralizing antibody titers by the Rapid Fluorescent Focus Inhibition Test (RFFIT) following the Centers for Disease Control and Prevention (CDC) protocol. Neutralizing antibody titers were expressed in IU per $\mathrm{mL}$. Subjects with antibody titers $\sim 0.5$ IU per $\mathrm{mL}$ were considered to have seroconverted. Such seroconversions served as the primary evaluation criteria for immunogenicity, while the Geometric Mean Concentrations (GMC) derived from the logarithmic transformation of the antibody titers obtained on days 7, 14 and 28 served as the secondary quantitative evaluation criteria. The significance of the GMC values was assessed in relation to their 95 percent confidence intervals $(95 \% \mathrm{CI})$.

\section{Subject withdrawal procedure}

When a subject failed to appear for a follow-up examination, efforts were undertaken to locate and determine the subject's health status. Subjects who were unable to return within the allowable time frame still completed their vaccination but were excluded from the immunogenicity analysis. However, their safety evaluation data were still considered. The subjects who decided to withdraw from the study were allowed to do so, but it was ascertained that the withdrawal was not due to an adverse event. The reasons for withdrawal were noted in the CRF.

\section{Data analysis}

Data recorded in the individual CRFs were encoded and analyzed using EPI-INFO version 6.0, Centers for Disease Control, USA.

\section{Results}

One hundred forty nine subjects were enrolled in this clinical trial, 73 in the pre-exposure group (Group 1 ) and 76 in the post-exposure group (Group 2). Eventually, 120 subjects (80\%), 60 in each group, completed the day 28 ID immunization and were included in the According-to-Protocol (ATP) analysis of immunogenicity. Twenty-nine subjects were withdrawn from the study mainly because of conflict with their guardian's work schedule. Three subjects withdrew because of failure to submit to blood extraction. However, the safety data from all the 149 immunized subjects on day 0 and those that could be obtained from days 3 and 7 were included in the intent to treat analysis.

The safety evaluation data is presented in Table 2. Mild local reactions, particularly redness and itchiness were observed in varying frequencies (4.0-63.0\%) among the subjects in both groups throughout the evaluations. Only one subject, at most, experienced mild pain at any evaluation point. No moderate or severe reactions were observed or reported by any of the subjects, including those who dropped out. Mild fever was observed in only one subject in Group 1 and two in Group 2, and it occurred only in association with the first day (day 0 ) of vaccine administration. No other systemic reaction was noted in any of the subjects during any of the evaluation points. There was no indication for administering treatment for any of the reactions noted.

All the 120 subjects included in the ATP analysis for immunogenicity demonstrated seroconversions (antibody titers were greater than the WHO cut-off level of $0.5 \mathrm{IU} / \mathrm{mL}$ ) on days 14 and 28 (Table 3). Thus, the seroconversion rates for both groups were $100 \%$, as applicable to these 120 subjects. Three subjects in Group I presented values greater than $60 \mathrm{IU} / \mathrm{mL}$. The GMC values for Group 1 were 3.30 $\mathrm{IU} / \mathrm{mL}$ and $4.37 \mathrm{IU} / \mathrm{mL}$ on days 14 and 28 respectively, while those of Group 2 were $3.73 \mathrm{IU} / \mathrm{mL}$ and 4.82 $\mathrm{IU} / \mathrm{mL}$, respectively. These GMC values were within their 95\% CI's (Table 4). 
Table 2. The number and percentage (\%) of subjects with mild reactions in the PVRV pre- and post-exposure ID regimen groups.

\begin{tabular}{|c|c|c|c|c|c|c|c|}
\hline \multirow[t]{2}{*}{ Reactions } & \multicolumn{3}{|c|}{ Pre-exposure group } & \multicolumn{4}{|c|}{ Post-exposure group } \\
\hline & Day 0 & Day 7 & Day 28 & Day 0 & Day 3 & Day 7 & Day 28 \\
\hline Starting $N=149(73 / 76)$ & $\mathrm{N}=73$ & $\mathrm{~N}=67$ & $\mathrm{~N}=60$ & $\mathrm{~N}=76$ & $N=63$ & $\mathrm{~N}=63$ & $\mathrm{~N}=60$ \\
\hline \multicolumn{8}{|l|}{$\begin{array}{l}\text { Local reactions } \\
\text { (injection site) }\end{array}$} \\
\hline Redness & $28(38.4 \%)$ & $6(9.0 \%)$ & $13(21.7 \%)$ & $3(3.9 \%)$ & $40(63.5 \%)$ & $10(15.9 \%)$ & $20(33.3 \%)$ \\
\hline Itchiness & $15(20.5 \%)$ & $16(23.9 \%)$ & $3(5.0 \%)$ & $8(10.5 \%)$ & $12(19.0 \%)$ & $21(33.3 \%)$ & $3(5.0 \%)$ \\
\hline Pain & $1(1 \%)$ & $1(1.5 \%)$ & 0 & 0 & $1(1.6 \%)$ & $1(1.6 \%)$ & 0 \\
\hline \multicolumn{8}{|l|}{ Systemic reactions } \\
\hline Fever & $1(1.4 \%)$ & 0 & 0 & $2(2.6 \%)$ & 0 & 0 & 0 \\
\hline Others & 0 & 0 & 0 & 0 & 0 & 0 & 0 \\
\hline
\end{tabular}

Table 3. The number and percentage (\%) of seroconversions as per titer level on days 14 and 28 in the PVRV pre- and postexposure ID regimen groups (Group 1 and 2).

\begin{tabular}{lllll}
\hline RFFIT titers in UI/mL & \multicolumn{2}{l}{ Pre-exposure group } & \multicolumn{2}{l}{ Post-exposure group } \\
\cline { 2 - 5 } & Day 14 & Day 28 & Day 14 & Day 28 \\
\hline & $\mathrm{N}=60$ & $\mathrm{~N}=60$ & $\mathrm{~N}=60$ & $\mathrm{~N}=60$ \\
$0.5-0.99$ & $4(6.7 \%)$ & $3(5.0 \%)$ & $1(1.7 \%)$ & $1(1.7 \%)$ \\
$1-30.99$ & $56(93.3 \%)$ & $54(90.0 \%)$ & $59(98.3 \%)$ & $57(95.0 \%)$ \\
$31-60.99$ & & & & $1(1.7 \%)$ \\
61 and up & & $3(5.0 \%)$ & & $1(1.7 \%)$ \\
\hline
\end{tabular}

Note: No subject had detectable antibodies on Day 0.

Table 4. The geometric mean concentrations (GMC, in IU/mL) and $95 \%$ confidence intervals ( $95 \% \mathrm{CI})$ in the PVRV pre- and post-exposure ID regimen groups.

\begin{tabular}{lllllll}
\hline Ab Assay points & Pre-exposure group & \multicolumn{4}{l}{ Post-exposure group } \\
\cline { 2 - 7 } & Number of subjects & GMC & $\mathbf{9 5 \%}$ CI & Number of subjects & GMC & 95\% CI \\
\hline Day 14 & 60 & 3.30 & $2.62-3.90$ & 60 & 3.73 & $3.11-4.47$ \\
Day 28 & 60 & 4.37 & $3.68-6.12$ & 60 & 4.82 & $3.90-5.97$ \\
\hline
\end{tabular}

\section{Discussion}

The occurrences of the mild local (redness and itchiness) and systemic (fever or body temperature rise) reactions in some of the subjects were considered as common sequels to any standard immunization regimens using current types of vaccines. The greater number of subjects that were observed to have developed redness at the site of vaccination in Group 1 compared with Group 2 was attributed to mere chance variation. Although, regardless of the cause, such mild redness (even with swelling) and mild itchiness at the site of injection is known to typically occur with most routine vaccinations including those for rabies [9]. These reactions are usually mild and are self-limiting.

The GMC values for both groups on days 14 and 28 were noted to have reached high levels. Notably, the GMC values in Group 1 were at levels close to the GMC values obtained in Group 2, even though the post-exposure subjects received additional vaccine doses with an additional vaccination on day 3 . This seems to signify that even a single $0.1 \mathrm{~mL}$ ID dose of the vaccine administered at three points (days 0,7 and 28) instead of four (plus a day 3 ) is sufficiently immunogenic. However, it is to be noted that six subjects in Group 1 had titers below $1 \mathrm{IU} / \mathrm{mL}$ on day 
14 and two with titers at just above the cut-off value $(0.5 \mathrm{IU} / \mathrm{mL})$ on day 28 , whereas in Group 2 there were only two subjects (one each on day 14 and 28) with titers below $1 \mathrm{IU} / \mathrm{mL}$. Nevertheless, all these titers signified adequate seroconversions, so a conclusion on comparative merits could not be drawn. Overall, both groups gained reasonable titer levels on day 14 , which signify a desirable rate of antibody development within two weeks of initiating immunization. The distributions of the subjects as per titer levels appear to be similar for both groups, except for a few outlying subjects, which accounted for the differences in the $95 \%$ CIs. Thus, both groups seem to have generally demonstrated adequate antibody responses with upsurges in GMCs from day 14 to day 28. However, day 7 and beyond day 28 titers were not determined in this study, which could otherwise further reveal relevant information on the rates and extents of seroconversions. As this study did not aim to determine the relative advantage of the (2-2-2-0-2) post-exposure ID regimen over the preexposure regimen, which requires fewer vaccine doses. This matter is set aside for further evaluation in the future.

The $20 \%$ dropout rate was believed to have had a minimal impact on the overall outcome of the study as the reasons given for withdrawal from the study were not related to occurrences of adverse reactions or to inadequate antibody responses. Based on the clinical observations and serologic assay results, it can be concluded that Abhayrab is a safe and immunogenic vaccine that could elicit the required, and even very high, antibody responses, if given intradermally to animal-bite patients. This study is in agreement with the report and recommendation of WHO stating that modern cell-culture rabies vaccines are well tolerated, safe and can be administered intradermally to people of all ages [8]. The conclusion is in line with the findings in a previous study conducted by the Indian Council of Medical Research involving four different human rabies vaccines administered to subjects employing the intradermal regimen. Those results revealed that out of the four vaccines studied, only three, including Abhayrab, were suitable for use in intradermal rabies vaccination in India, with Abhayrab eliciting $100 \%$ seroconversion rates on days 14,28 , and 90 , and the highest geometric mean antibody titers (GMT) on days 28 and 90 [10]. For more than 20 years, the ID route has been routinely and effectively used for other rabies vaccines
[11-13], and recently for some other important human vaccines [14-18]. The use of the ID route, coupled with the use of a relatively affordable vaccine product, leads to significant savings in the total amount of vaccines required for a full pre- or post-exposure vaccination series, thereby enormously reducing the cost of active immunization for the benefit of the public.

In conclusion, Abhayrab is a safe and immunogenic rabies vaccine when administered intradermally. As this study involved healthy volunteer subjects, a follow-on study involving category III dogbite patients is being planned with both the test vaccine and a rabies immunoglobulin (RIG) being administered to the patients to determine the effect of RIG on the active antibody stimulation expected from the vaccine.

\section{Acknowledgement}

This study received financial support from the Human Biological Institute of Ooty, India. None of the authors has any conflicts of interest to report.

\section{References}

1. PSMD Task Force Recommendation for Rabies Pre and Post exposure prophylaxis, Phil J Microbiol Infect Dis. 1997, 26:25-9.

2. WHO Expert Committee on Rabies, 8th report, TRS 824, World Health Organization, Geneva, 1992.

3. World Health Organization, Report of a WHO consultation on intradermal application of human rabies vaccines, Geneva, 13-14 March 1995.

4. Matha IS, Salunke SR. Immunogenicity of purified vero cell rabies vaccine used in the treatment of fox-bite victims in India, CID. 2005; 40:611-3.

5. Sampath G, Sangram P, Ananda MN, Udaya Bhaskar RY, Palaniappan C, Suhasini RV. Clinical study to assess the safety and immunogenicity of vero cell culture inactivated rabies vaccine, Journal of APCRI (Assoc Prevent Contral Rabies India). 2000; 1:50-2.

6. Sampath G, Suhasini RV, Lakshmi PR, Udayabhaskara RY, Palaniappan C. An immunogenicity study of a newly introduced purified vero cell rabies vaccine (Abhayrab) manufactured in India. Vaccine. 2005; 23: 897-900.

7. Kempegowda Institute of Medical Sciences. A Guide to Intradermal Rabies Vaccination. Bangalore, India: Website: www.kimscommunitymedicine.org, 2006.

8. WHO recommendations on rabies post-exposure treatment and the correct technique of intradermal immunization against rabies. Geneva: WHO, 1997. 


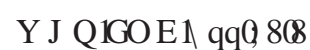

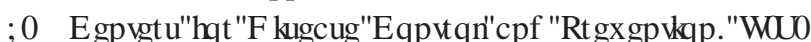

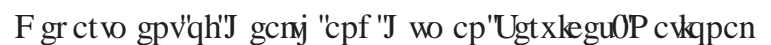


\%DMFVDCG\&RP P RQ4 XHWRRQVIBRWEOH6IGHHIFW

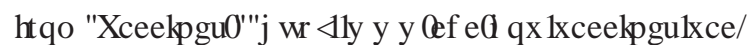

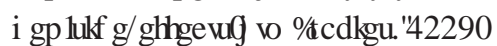

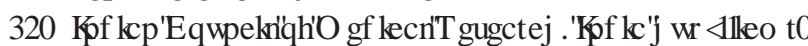

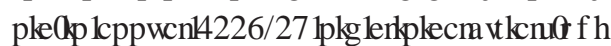

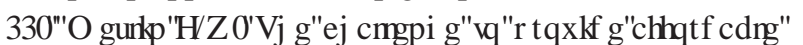

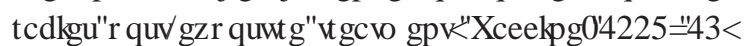
परिपाए

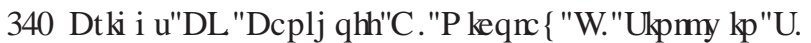

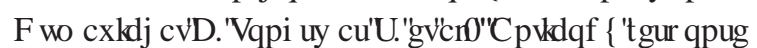
RIDSDWHQWDUMUSRWWH SRVXUIUEIHM YDFFLQDRQ Z LUKNP DOUNOCHP DORRVHRIISXUIHCHFIFNHP EU R

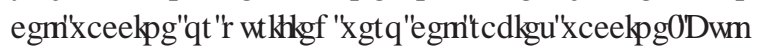

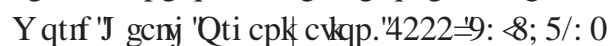

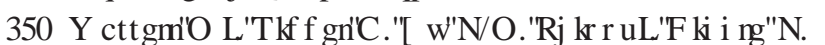

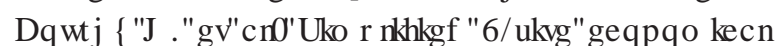
IQNOCHP DOSRWMH SRVXIHIDEIHNYDFFLHIHI IP HQID WOQRP L] HGFRQNRCHGFRP SDIMRQZZ WW WMQGDG



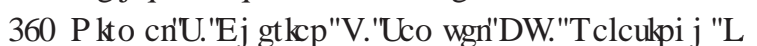

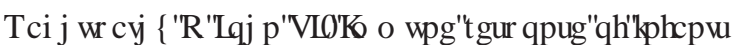
WRIIUFWRQDOGRUHVRIIQMDGHP DOOIDCP IQLWMHG

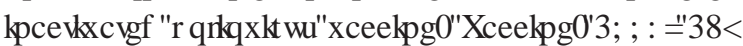

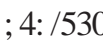

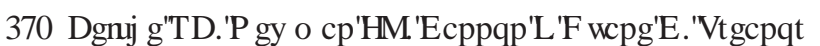
- 9 9DQ + RHFNH\&DHMDO 6HXX IDQNERG IUHSRQMH DUMUIQNOCHP DOYLFFLDWRQD DQMWQIXHD DII 1

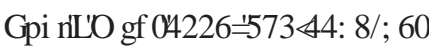

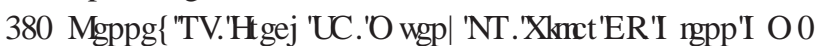
' RUHISDUQ IZ LWRIQNDCHP DOIQYAFWRQRRIIQIOXHD] D

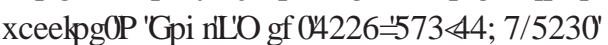

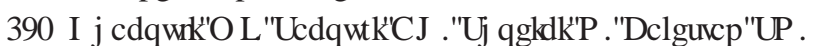

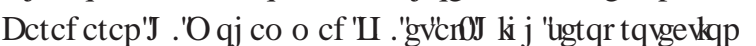
UMMLQGXFHGE IIQMDGHP DODGP IQLUWURQ RIDD

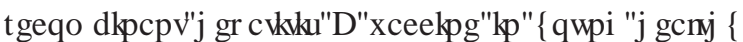
DGXONGFRP SDUMRQZ LMR WWQGDCGLQMP XVEXOU

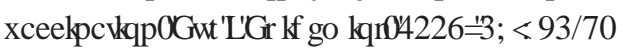

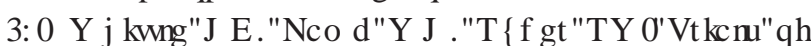

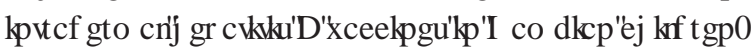

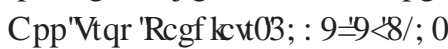

